\title{
Superluminal travel requires negative energies
}

\author{
Ken D. Olum* \\ Institute of Cosmology \\ Department of Physics and Astronomy \\ Tufts University \\ Medford, MA 02155
}

(August 1998)

\begin{abstract}
I investigate the relationship between faster-than-light travel and weakenergy-condition violation, i.e., negative energy densities. In a general spacetime it is difficult to define faster-than-light travel, and I give an example of a metric which appears to allow superluminal travel, but in fact is just flat space. To avoid such difficulties, I propose a definition of superluminal travel which requires that the path to be traveled reach a destination surface at an earlier time than any neighboring path. With this definition (and assuming the generic condition) I prove that superluminal travel requires weak-energycondition violation.
\end{abstract}

04.20.Gz

*Email address: kdo@alum.mit.edu 
A longstanding question asks whether the metric of spacetime can be manipulated to allow very rapid travel between spatially distant points. (I will call this "superluminal" or "faster than light" even though, of course, I'm not proposing to go faster than a light signal in the same metric). If one allows arbitrary states of matter, one can construct such spacetimes, as in the examples of Alcubierre [1] and Krasnikov [2,3]. However, these spacetimes require negative energy densities [3,4]; i.e., they violate the weak energy condition (WEC), which states that $T_{\mu \nu} V^{\mu} V^{\nu} \geq 0$ for any timelike vector $V^{\mu}$. The question then is whether it is possible to have superluminal travel without this violation.

To answer this question one must first specify what one means by "superluminal travel." The general idea is that some modification of the metric allows signals to propagate between two spacetime points that otherwise would be causally disconnected. However, it may not always be easy to distinguish such superluminal travel from a situation in which the supposedly distant object has been brought nearby, so that travel at ordinary speeds allows one to reach it in a short time.

As a concrete example consider a spacetime with metric

$$
d s^{2}=\left(-1+4 t^{2} x^{2}\right) d t^{2}-4 t x\left(1-t^{2}\right) d x d t+\left(1-t^{2}\right)^{2} d x^{2}
$$

in the region $-1<t<1$. Null rays in this metric have

$$
\frac{d x}{d t}=\frac{ \pm 1+2 t x}{1-t^{2}}
$$

and, for example, a right-going null geodesic from the origin has $x=t /\left(1-t^{2}\right)$ as shown in Fig. 1. It would appear that this metric allows superluminal travel. Starting from the origin one can reach points at arbitrarily large $x$ in time $t<1$. If the earth were fixed at $x=0$ and a distant star at $x=1$, we could travel from the earth at $t=0$ to the star in time $(1+\sqrt{5}) / 2 \approx 0.618$.

However, this metric has nothing to do with superluminal travel. It is just flat space with an odd choice of coordinates: if we let $x^{\prime}=x\left(1-t^{2}\right)$ the metric becomes $d s^{2}=-d t^{2}+d x^{\prime 2}$. The star which is "fixed" at $x=1$ is in fact traveling on a path which brings it closer to the earth. The motion of the destination, rather than any superluminal travel, is what reduces the time to reach the star.

The point of this example is that just examining a metric may not easily reveal whether it would be reasonable to regard the spacetime as containing superluminal travel. One must have some idea how to distinguish bringing a place closer from establishing an arrangement which allows one to travel there more quickly.

In some simple cases, however, the spacetime is flat, except for a localized region not including the points between which one wishes to travel. Then there is no question about the distance between the two points, because they lie in a single region of Minkowski space. The Alcubierre bubble [1] and the Krasnikov tube [2,3] are of this type if one imagines the tube to be finite in length or the bubble to exist for a finite time. A simple example of this sort is shown in Fig. 2. The flat metric has been modified in such a way that there is a causal path $P$ from $\left(t_{1}, x_{1}\right)$ to $\left(t_{2}, x_{2}\right)$ even though $x_{2}-x_{1}>t_{2}-t_{1}$. Since there is a connected region of Minkowski space which includes $\left(t_{1}, x_{1}\right)$ and $\left(t_{2}, x_{2}\right)$, it is well-defined to say that the interval between these points would be spacelike without the modification to the metric. 


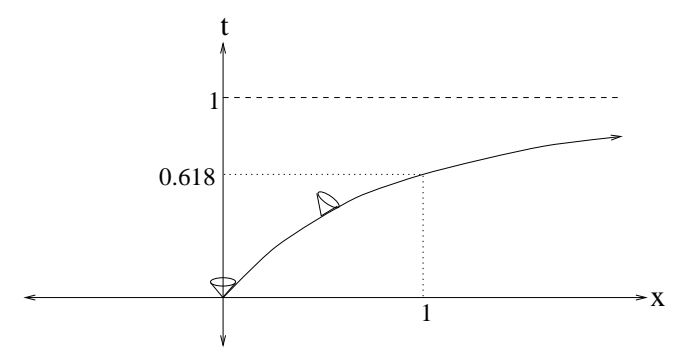

FIG. 1. A null geodesic in the metric of Eq. (11). It appears that one can reach arbitrary distances before $t=1$.

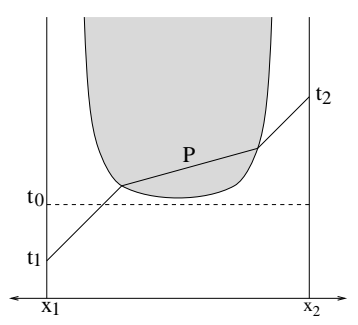

FIG. 2. Superluminal travel is produced by modifying the shaded region of Minkowski space. The modification is localized between $x_{1}$ and $x_{2}$ and after $t_{0}$. Because of this modification, there is a causal path $P$ connecting $\left(t_{1}, x_{1}\right)$ to $\left(t_{2}, x_{2}\right)$, even though $x_{2}-x_{1}>t_{2}-t_{1}$.

In this simple case we can show that WEC must be violated, using the existing theorems [5] that prohibit closed timelike curves. Let $S$ be a spacetime that is flat except for a region with $t>t_{0}, x \in\left[x_{1}, x_{2}\right], y \in\left[y_{1}, y_{2}\right]$, and $z \in\left[z_{1}, z_{2}\right]$, and suppose there is a causal path $P$ that connects points $\left(t_{1}, x_{1}, y_{0}, z_{0}\right)$ and $\left(t_{2}, x_{2}, y_{0}, z_{0}\right)$ with $t_{2}-t_{1}<x_{2}-x_{1}$. Suppose also that $S$ contains no singularities and that the modified region of $S$ obeys the generic condition [8], i.e., each null geodesic that passes through that region contains a point where $K_{[a} R_{b] c d[e} K_{f]} K^{c} K^{d} \neq 0$, where $K$ is the tangent vector to the geodesic. Let $\Delta t=t_{2}-t_{1}$. Consider a new spacetime $S^{\prime}$ which consists of the portion of $S$ between $x_{1}$ and $x_{2}$ with the same metric as $S$, and with points $\left(t, x_{1}, y, z\right)$ and $\left(t+\Delta t, x_{2}, y, z\right)$ identified for each $t, y$, and $z$. In $S^{\prime}$, the path $P$ is a closed causal curve. However, causal paths that travel only through the flat part of $S^{\prime}$ cannot be closed, because $\Delta t<x_{2}-x_{1}$. In particular no point with $t<t_{0}-\Delta t$ can be on a closed causal path. So there is a Cauchy horizon in $S^{\prime}$ in the future of the surface $t=t_{0}-\Delta t$ and in the past of (or at) the path $P$. If $S$ has no singularities, than $S^{\prime}$ will not have any either. Thus by Tipler's and Hawking's theorems [5] [7], WEC must be violated somewhere in $S^{\prime}$. Since WEC is a local condition, it must also be violated at the corresponding point of $S$.

In a general spacetime we need a definition of superluminal travel. Here I propose the following idea: a superluminal travel arrangement should have some path along which it functions best. A signal propagating along this best path should travel further than a signal on any nearby path in the same amount of (externally defined) time. To formalize this we construct small spacelike 2-surfaces around the origin and destination points and say 


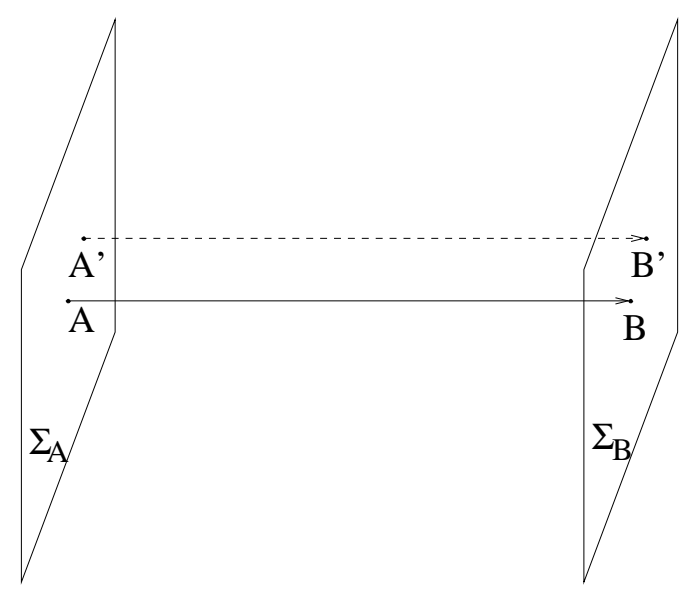

FIG. 3. A superluminal travel arrangement. The metric has been so arranged so that a causal path (solid line) exists between $A$ and $B$ but there are no other causal paths (such a possibility is shown dashed) that connect the 2 -surfaces $\Sigma_{A}$ and $\Sigma_{B}$.

that while the destination is reachable from the origin, no other point of the destination surface is reachable from any point of the origin surface. See Fig. 3. Of course this would be trivial if, for example, the destination surface were curved in such a way that the destination were merely the closest point on its surface to the origin. To avoid this problem we require that the origin (destination) surface be composed of a one-parameter family of spacelike geodesics through the origin (destination) point. Formally, we say that a causal path $P$ is superluminal from $A$ to $B$ only if it satisfies

Condition 1 There exist 2-surfaces $\Sigma_{A}$ around $A$ and $\Sigma_{B}$ around $B$ such that (i) if $p \in \Sigma_{A}$ then a spacelike geodesic lying in $\Sigma_{A}$ connects $A$ to $p$, and similarly for $\Sigma_{B}$, and (ii) if $p \in \Sigma_{A}$ and $q \in \Sigma_{B}$ then $q$ is in the causal future of $p$ only if $p=A$ and $q=B$.

This condition might not be sufficient for what one would call superluminal travel, because it is possible that while $P$ arrives earlier than any nearby path, it is still slower than a path some larger distance away. In this case, we would not want to say that $P$ was superluminal.

Suppose that there is a path $P$ satisfying the above condition, and suppose also that the generic condition [8] holds on $P$. The generic condition holds whenever there is any normal matter or any transverse tidal force anywhere on $P$. With these assumptions, we will show that WEC must be violated at some point of $P$.

First we note that $P$ must be a null geodesic. If $P$ is not a geodesic it can be varied to make a timelike path from $A$ to $B$. If $P$ is timelike anywhere, then it can be varied to make a timelike path from $A$ to points of $\Sigma_{B}$ other than $B$.

Let $K$ be the tangent vector to the geodesic $P$. The vector $K$ must be normal to the surface $\Sigma_{A}$. Otherwise there would be points on $\Sigma_{A}$ in the past of points on $P$. Similarly, $K$ must be normal to $\Sigma_{B}$.

Now define a congruence of null geodesics with affine parameter $v$, normal to $\Sigma_{A}$, and extend $K$ to be the tangent vector at each point of the congruence. 
Could there be some point $x \in P$ that is conjugate to the surface $\Sigma_{A}$ ? If $x$ were an interior point of $P$ then it would be possible to deform $P$ into a timelike path. If $x=B$ then different geodesics of the congruence would all end at $B$ or points very near to $B$. These geodesics would have different tangent vectors, which could not all be normal to $\Sigma_{B}$. Thus no point on $P$ is conjugate to $\Sigma_{A}$.

Now we look at $\hat{\theta}$, the expansion of the geodesic congruence. It is given by $\hat{\theta}=K_{; m}^{m}$, where $m$ runs over two orthogonal directions normal to $K$. (All choices of such directions give the same $\hat{\theta}$.) At $A$ we use directions that lie in $\Sigma_{A}$ and at $B$ we use directions that lie in $\Sigma_{B}$. Since $\Sigma_{A}$ is extrinsically flat at $A$, the geodesics are initially parallel, so $\hat{\theta}=0$ at $A$. The evolution of $\hat{\theta}$ is given by the Raychaudhuri equation for null geodesics,

$$
\frac{d \hat{\theta}}{d v}=-R_{a b} K^{a} K^{b}+2 \hat{\omega}^{2}-2 \sigma^{2}-\frac{1}{2} \hat{\theta}^{2}
$$

where $\hat{\omega}$ is the vorticity, which vanishes here, $\hat{\sigma}$ is the shear, and $R_{a b}$ is the Ricci curvature tensor. Since there are no conjugate points, $\hat{\theta}$ is well-defined all along $P$. If the weak energy condition is satisfied, then $R_{a b} K^{a} K^{b} \geq 0$, so $d \hat{\theta} / d v \leq 0$. From the generic condition, $\hat{\sigma}$ cannot vanish everywhere, thus WEC implies

$$
\hat{\theta}<0
$$

at $B$. If we can show that instead $\hat{\theta} \geq 0$ at $B$, then WEC must be violated on $P$.

First we establish a basis for vectors at $B$. Let $E_{1}$ and $E_{2}$ be orthonormal vectors tangent to $\Sigma_{B}$ at $B$. Let $E_{3}$ be a unit spacelike vector orthonormal to $E_{1}$ and $E_{2}$ with $g\left(K, E_{3}\right)>0$. Let $E_{4}$ be the unit future-directed timelike vector orthogonal to $E_{1}, E_{2}$, and $E_{3}$. Using these vectors establish (Riemannian) normal coordinates near $B$. The space $\Sigma_{B}$ consists of the points with $t=z=0$.

Let $\lambda(s)$ be a smooth curve on $\Sigma_{A}$ with $\lambda(0)=A$. Let $\lambda(s, v)$ be the point an affine distance $v$ along the null geodesic from $\lambda(s)$. Eventually each geodesic will pass near $B$ and will cross the hypersurface with $t=0$. Call this crossing point $\lambda^{\prime}(s)$ and adjust the length of the vectors $K$ on $\Sigma_{A}$ so that $\lambda(s, 1)=\lambda^{\prime}(s)$. See Fig. 1 .

The $z$ coordinate of $\lambda^{\prime}(s)$ must be negative. Otherwise, points on $\Sigma_{B}(z=t=0)$ would be the future of points of the geodesics from $\Sigma_{A}$.

Let $Z$ be the tangent vector to $\lambda(s, v)$ in the $s$ direction. By construction, $K^{a} Z_{a}=0$ on $\Sigma_{A}$. This product is constant along each geodesic [8], so $K^{a} Z_{a}=0$ everywhere. If we follow along $\lambda^{\prime}(s)$ from $B$ we see that

$$
0=\frac{d}{d s}\left(K^{a} Z_{a}\right)=\left(K^{a} Z_{a}\right)_{; b} Z^{b}=K_{; b}^{a} Z_{a} Z^{b}+K^{a} Z_{a ; b} Z^{b} .
$$

The only non-vanishing components of $K$ are $K^{3}$ and $K^{4}$. Since $\lambda^{\prime}(s)$ lies in the $t=0$ hypersurface, $Z^{4}=0$ everywhere, so only $a=3$ contributes to $K^{a} Z_{a ; b}$ at $B$. Thus from Eq. (可)

$$
K_{; b}^{a} Z_{a} Z^{b}=-K^{3} Z_{3 ; b} Z^{b} .
$$

At $B, Z_{3}=0$. We must also have $Z_{3 ; b} Z^{b} \leq 0$ because otherwise $\lambda^{\prime 3}$ would become positive. By construction, $K^{3}>0$, so $K^{3} Z_{3 ; b} Z^{b} \leq 0$ and 


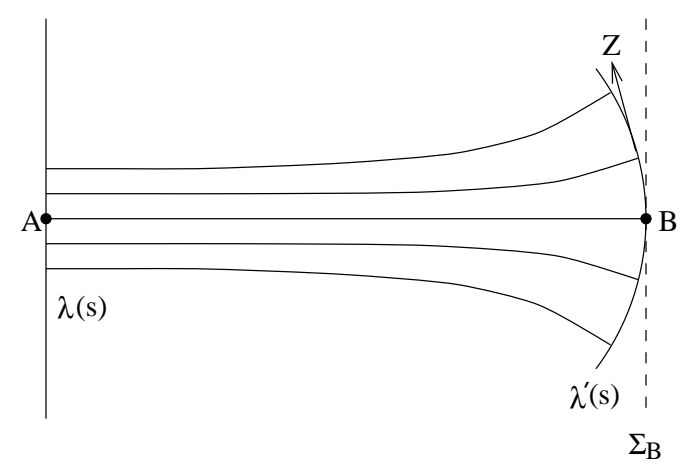

FIG. 4. Congruence of null geodesics from $\lambda(s)$ followed into the future until they reach points near $B$ with $t=0$ at a curve $\lambda^{\prime}(s)$ with tangent vector $Z$. At points near $B, \lambda^{\prime}(s)$ must have negative $z$ coordinate.

$$
K_{; b}^{a} Z_{a} Z^{b} \geq 0 .
$$

The congruence of geodesics provides a map from tangent vectors to $\lambda(s)$ at $A$ to tangent vectors to $\lambda^{\prime}(s)$ at $B$. Since there are no conjugate points, this map is non-singular and can be inverted. Thus we can find choices of $\lambda(s)$ that make $Z=E_{1}$ or $Z=E_{2}$. Then we find that $K_{; 1}^{1} \geq 0$ and $K_{; 2}^{2} \geq 0$ and so

$$
\hat{\theta}=K_{; m}^{m} \geq 0
$$

in contradiction to Eq. (44).

Thus we see that any spacetime that admits superluminal travel on some path $P$ (and thus, according to our definition, that satisfies Condition 1) and that satisfies the generic condition on $P$, must also violate the weak energy condition at some point of $P$.

One can compare this theorem with those of Tipler [5,6] and Hawking [7] that we used earlier. These theorems rule out the construction of closed timelike curves (CTC's) from a compact region unless there is WEC violation or a singularity on the boundary of the causality violating region. The present theorem rules out the existence, rather than construction, of superluminal travel, unless there is weak energy condition violation. Spacetime singularities do not provide an alternative (other than by making the purported path not actually reach the destination), and the WEC violation must occur along the path to be traveled.

This raises the question of whether the present theorem can be extended to rule out more time machines than the theorems of Tipler and Hawking do. However, this extension is not easily accomplished. Inside a CTC-containing region, each point will be in the future of each other point. Thus one cannot construct surfaces $\Sigma_{A}$ and $\Sigma_{B}$ with the required properties. Even if one puts the points $A$ and $B$ on the Cauchy horizon, it is still not possible to construct spacelike 2-surfaces that do not intersect the CTC-containing region.

Does this theorem mean that superluminal travel is impossible? No, because the weak energy condition is not obeyed by systems of quantum fields. The best example is the Casimir effect, and in fact, the Casimir effect does provide an example which satisfies condition 1.

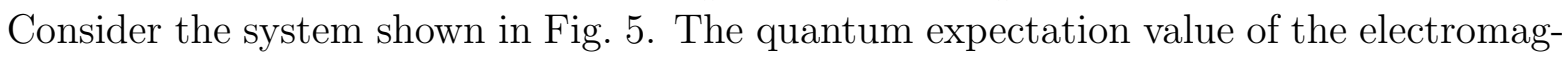




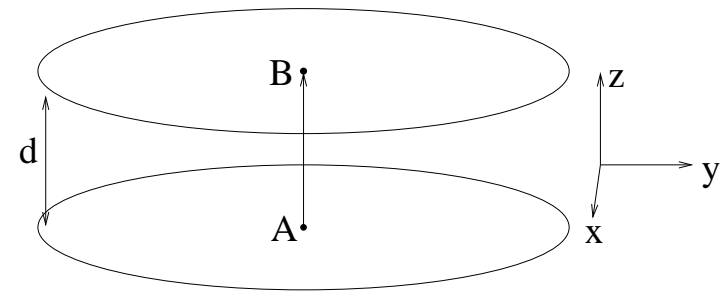

FIG. 5. Circular conducting plates give rise to a negative pressure and energy density, and a consequent advancement of the time of arrival of a null ray from $A$ to $B$.

netic stress-energy tensor between the plates is

$$
T_{a b}=\frac{\pi^{2}}{720 d^{4}} \operatorname{diag}(-1,1,1,-3) .
$$

For a geodesic traveling in the $z$ direction, we find

$$
R_{a b} K^{a} K^{b}=-\frac{2 \pi^{3}}{45 d^{4}}
$$

Now let $\Sigma_{A}$ be the lower plate and $\Sigma_{B}$ be the upper plate, and we can go through the argument above in reverse. We start with $\hat{\theta}=0$ as before, and now $\hat{\sigma}=0$ by symmetry. As before, $\hat{\omega}=0$, so the Raychaudhuri equation (3) gives

$$
\frac{d \hat{\theta}}{d v}=-R_{a b} K^{a} K^{b}>0
$$

so the geodesics around $P$ are defocused. Thus the geodesic $P$ travels further in the $z$ direction by the same $t$ than neighboring geodesics, and condition 1 is satisfied.

It is not clear whether this phenomenon is sufficient to provide a system of superluminal travel. The discussion above is not complete, because it does not account for the mass of the plates or of the supporting structure required to keep them apart against the tension in the field. A long, straight path traveling through the center of the plates arrives earlier than nearby paths, but it is possible that a path that avoids the system of plates entirely might arrive still earlier.

I would like to thank Arvind Borde, Allen Everett, Larry Ford, Michael Pfenning, and Tom Roman for helpful conversations. This work was supported in part by funding provided by the National Science Foundation.

Note added in proof: While this paper was in press, I learned of unpublished work by R. Penrose, R. D. Sorkin and E. Woolgar (gr-qc/9301015) which also discusses the connection between WEC violation and geodesic advancement. 


\section{REFERENCES}

[1] M. Alcubierre, Class. Quantum Grav. 11, L73 (1994).

[2] S. V. Krasnikov, Phys. Rev. D 57, 4760 (1998), gr-qc/9511068.

[3] A. Everett and T. A. Roman, Phys. Rev. D 56, 2100 (1997), gr-qc/9702049.

[4] M. J. Pfenning and L. H. Ford, Class. Quantum Grav. 14, 1743 (1997), gr-qc/9702026.

[5] F. J. Tipler, Phys. Rev. Lett. 37, 879 (1976).

[6] F. J. Tipler, Ann. Phys. 108, 1 (1977).

[7] S. W. Hawking, Phys. Rev. D 46, 603 (1992).

[8] S. W. Hawking and G. F. R. Ellis, The Large Scale Structure of Space-time (Cambridge University Press, London, 1973). 